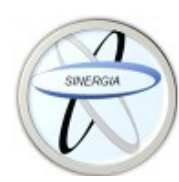

Revista Médica Sinergia

Vol. 6, Núm. 11, noviembre 2021, $\underline{\mathrm{e} 733}$

\title{
Dolor agudo en el servicio de urgencias
}

\section{Acute pain in the emergency department}

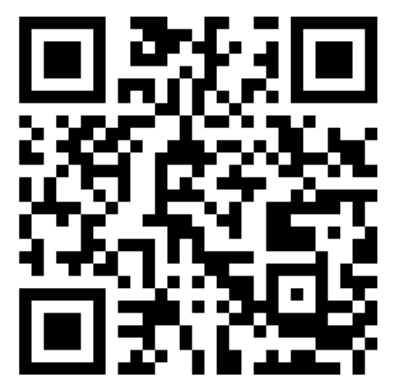

Recibido
$27 / 09 / 2021$

\author{
${ }^{1}$ Dra. Francela Isabel Diaz Mena \\ Hospital San Rafael de Alajuela, Alajuela, Costa Rica \\ (iD) https://orcid.org/0000-0002-7430-2816 \\ ${ }^{2}$ Dra. Ariadna Jazmín Flores Castro \\ Hospital Max Peralta Jiménez, Cartago, Costa Rica \\ (1) https://orcid.org/0000-0002-4826-5249
}

\section{RESUMEN}

El dolor en el servicio de urgencias se ha estudiado desde hace algún tiempo debido a la cantidad de consultas por esta causa. La respuesta fisiológica del dolor es producto del daño tisular que se experimenta, generando una respuesta química produciendo un impulso eléctrico, para finalmente percibir el dolor. Durante el abordaje en el servicio de urgencias es importante realizar una historia clínica completa y detallada con material de apoyo necesario. Actualmente el manejo del dolor debe ser visto de manera multidisciplinaria tanto desde el punto de vista farmacológico y no farmacológico. Siendo los AINES los medicamentos de primera línea para el manejo del dolor agudo, esto dependiendo de la etiología del dolor, en la presente revisión bibliográfica nos enfocamos en los medicamentos más utilizados y disponibles en nuestros centros de salud pública.

PALABRAS CLAVE: dolor; emergencias; clasificación; diagnóstico; tratamiento.

\section{ABSTRACT}

Pain in the emergency department has been studied for some time due to the number of consultations for this cause. The physiological response of pain is the product of the tissue damage that is experienced, generating a chemical response producing an electrical impulse, to finally perceive the pain. During the emergency department approach, it is important to take 
a complete and detailed medical history with the necessary support material. Currently, pain management must be seen in a multidisciplinary way, both from a pharmacological and nonpharmacological point of view. Since NSAIDs are the first-line drugs for the management of acute pain, depending on the etiology of the pain, in this literature review we focus on the drugs most used and available in our public health centers.

KEYWORDS: pain; emergencies; classification; diagnosis; treatment.

${ }^{1}$ Médica general, graduada de la Universidad Latina de Costa Rica (U. Latina). Cód. MED16186. Correo: francelafd91@gmail.com

${ }^{2}$ Médica general, graduada de la Universidad Autónoma de Centroamérica (UACA). Cód. MED16304. Correo: ariadna.flc@gmail.com

\section{INTRODUCCIÓN}

La asociación internacional del dolor lo define como una experiencia sensorial y emocional desagradable asociado a daño tisular real o potencial. Se genera producto de múltiples mecanismos fisiológicos en respuesta a un estímulo, el cual es percibido en cada individuo de manera personal, lo que lo hace tener un manejo más complejo. El dolor comprende casi el $80 \%$ de las causas de derivación al departamento de urgencias (3). El manejo en el servicio de urgencias de manera eficiente y segura es considerado como una piedra angular debido a que es la principal causa de consulta. Los médicos que trabajan en este servicio deben de reconocer y valorar el dolor tanto agudo como el crónico de manera empática, comprendiendo el sufrimiento que este conlleva, además brindar un abordaje multimodal al paciente (3). El costo económico es elevado solamente en Estados Unidos de Norteamérica, en el 2010, se estiman gastos totales entre $\$ 560$ y $\$ 635$ billones de dólares, siendo más altos los costos por dolor que los generados por otras enfermedades, como la cardiaca (\$309 billones), cáncer ( $\$ 243$ billones) y diabetes (\$188 billones) (4). Además, el dolor es la principal causa de ausentismo laboral, lo que conlleva a generar mayor costo secundario.
Es importante conocer la etiología del dolor, y la duración de este, según las características podemos clasificarlo como leve, moderado o severo.

El fin de la presente revisión bibliográfica es lograr en primera instancia se valore el dolor de manera adecuada enfatizando en la importancia que este genera en el estado de salud del paciente, y además brindar herramientas para ser abordarlo de una manera integral $(4,8)$.

\section{MÉTODO}

Se realizó una revisión bibliográfica de artículos recientes, desde el año 2017 hasta el 2021, tanto en inglés como en español, con un total de 19 , se eligieron basados en la satisfacción de los objetivos de revisión, y respaldos por revistas científicas destacadas.

La búsqueda de dicha literatura se realizó mediante múltiples bases de datos como: ELSEVIER, Medline, Medigraphic, Scielo y revistas científicas.

\section{DEFINICIÓN DEL DOLOR}

El dolor según la International Asociation for the Study of Pain (IASP) se define como una experiencia sensorial 0 emocional desagradable, asociada a daño tisular real o potencial (4). El dolor es uno de los 
principales motivos de consulta de los pacientes remitidos al hospital y comprende casi el $80 \%$ de las causas de referencia al servicio de urgencias (1). El Colegio Americano de Médicos de Emergencia buscan estrategias para mejorar el manejo del dolor agudo para los pacientes que consultan al servicio de urgencias, ya que se debe reconocer la necesidad de un adecuado abordaje del dolor para lograr un tratamiento rápido, seguro y eficaz (1).

El manejo óptimo del dolor agudo es específico para cada paciente y se debe valorar de acuerdo con factores como raza, edad, sexo, capacidad para expresar el dolor y enfermedades concomitantes. Inicialmente se debe conocer las características del dolor para clasificarlo en agudo, crónico, y debido al cáncer $(1,2)$.

\section{FISIOLOGÍA}

Existen cuatro mecanismos básicos en la producción del dolor: transducción, conducción, modulación y percepción.

Transducción: ante un estímulo físico, térmico o químico se liberan múltiples neurotransmisores como prostaglandinas $(P G)$, bradicinina $(B K)$, factor de necrosis tumoral alfa (TNF-a), hidrogeniones $(H)$, factor de crecimiento neural (NGF), histamina, ATP y de manera retrograda sustancia $P(S p)$ y péptido relacionado con el gen de la calcitonina (CGRP) que producen vasodilatación y degranulación de mastocitos esto se traduce en una señal eléctrica al incrementar la expresión de canales de sodio generando la transmisión del impulso, este es percibido por los nociceptores, posteriormente la señal es conducida por fibras nerviosas, principalmente tipo A-delta y $\mathrm{C}$, proceso de conducción (4), seguidamente ocurre proceso el de modulación, este consiste en el ingreso del impulso hacia las astas posteriores donde se encuentran células inhibitorias gabaérgicas y células de la glía que tienen función excitatorias. El producto de este proceso de modulación es el aumento o bien el bloqueo del impulso, que viajará por los tractos espinotalámicos hacia el tálamo y parte del sistema límbico para finalmente llegar a la corteza somatosensorial y así percibir el dolor. Además, en la corteza y tallo cerebral existen también impulsos descendentes inhibitorios mediados por adrenalina y serotonina con el objetivo de disminuir el estímulo percibido por los nociceptores por tanto se hace un bloqueo parcial del dolor de manera fisiología (4).

\section{CLASIFICACIÓN DEL DOLOR}

La clasificación del dolor va a depender de la fisiología de este (5). Al ser una sensación subjetiva se han creado múltiples escalas para valorar el dolor de manera individual, existe la escala numérica la cual consiste en describir la intensidad del dolor con el numero 1 si es sumamente leve y 10 que es el peor dolor que ha experimentado el paciente. Existe también la gráfica de expresión verbal, facial entre otras. Es importante que al evaluar el dolor debemos utilizar un adecuado instrumento, que se adapte a la edad del paciente y problemas cognitivos (6).

Dolor agudo: se caracteriza por ser de duración menor de 30 días y disminuye con el tiempo, puede tener causa conocida o no, un ejemplo de este es el dolor por trauma, fracturas de huesos, apendicitis, quemaduras y dolor durante procedimientos (2). En algunas ocasiones se puede acompañar clínicamente de taquicardia, hipertensión, diaforesis esto causado por la descarga adrenérgica asociada. 
Dolor crónico: Tienen una duración mayor de 30 días, puede ser de manera continua o en intervalos durante meses o años. En algunos casos, hay exacerbaciones agudas del dolor crónico. Entre estos está: lumbalgia, neuropatía diabética, neuralgia, esclerosis múltiple, dolor fantasma entre otros

También podemos clasificar el dolor según la etiología de este:

Nociceptivo: el cual es causado por un estímulo químico, mecánico o térmico a nivel visceral o somático.

Neuropático: este se origina por daño o estímulo neuronal, se caracteriza por ser persiste y no desaparece a pesar de que el estímulo no continúe presente (11).

Crisis aguda en una condición dolorosa recurrente: ese tipo de dolor puede aparecer en cualquier momento, por enfermedades crónicas de causa no maligna, por ejemplo, células falciformes, migraña y fibromialgia entre otras.

\section{DIAGNÓSTICO}

Para realizar un adecuado diagnóstico se debe valorar la severidad del dolor, no por el pronóstico de la enfermedad del paciente. La evaluación del dolor incluye: anamnesis, examen físico completo y ampliar con

Figura 1. Escalas para valoración del dolor

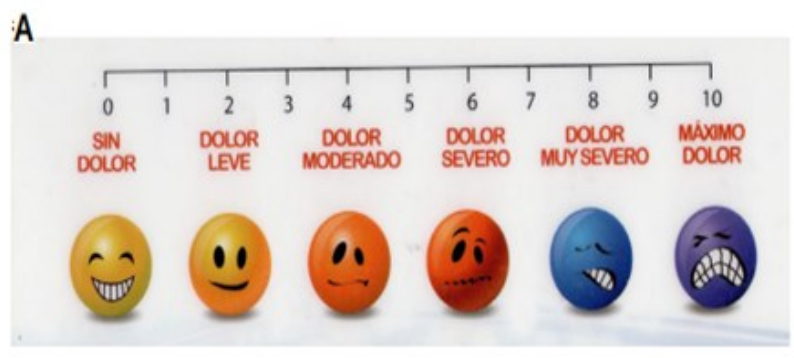

B

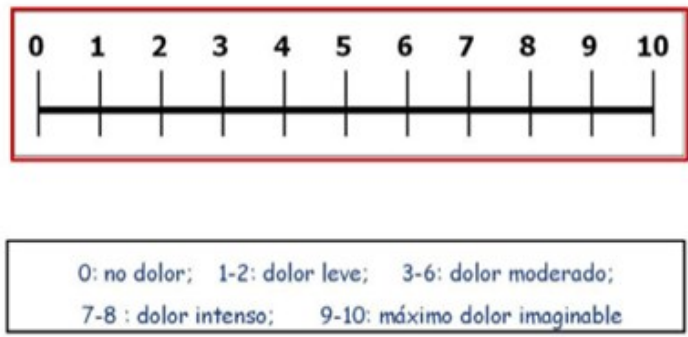

Fuente. Figura a obtenida desde Dolor COMEDEM C. Clínica del dolor COMEDEM. clinicadeldolorcomedem.com, figura b obtenida desde: Hospital Denens http://blogcas.hospitaldenens.com 
Bloquean el enzima ciclooxigenasa (COX) de forma no selectiva reversible que tiene las isoenzimas: COX-1, COX-2 y por tanto la síntesis de prostaglandinas. Se recomienda prescribir inicialmente con la dosis más baja, para dolores leves a moderados (11). Están disponibles en diversas vías: oral, intravenosa, rectal, sublingual, intramuscular o tópica. Podemos mencionar entre los más utilizados Ibuprofeno, sulindaco y diclofenaco, son similares en su efecto analgesico y mecanismos de acción. Los antiinflamatorios no esteroideos (AINEs) convencionales y COX-2 selectivos no se recomiendan como tratamiento de primera línea en adultos mayores, especialmente cuando hay polifarmacia o comorbilidades. En este caso, lo ideal sería iniciar con paracetamol a dosis plena (4).

- Paracetamol: es un analgésico y antipirético que inhibe la síntesis de prostaglandinas únicamente a nivel central. Si bien no es la más potente de las opciones, si es la más segura (4). A pesar de ellos la sobredosis por paracetamol es la principal causa de insuficiencia hepática aguda medicamentosa en Estados Unidos. El daño es producido por el metabolito tóxico N-acetil-p-benzoquinona (NAPQI) (15). Si tiene potencial de hepatotoxicidad, por lo que su dosis se limita a 4 gramos al día en pacientes que no ingieren alcohol ni otros medicamentos que tengan hepatotoxicidad (4).

- Ibuprofeno: el ácido 2-(4-isobutilfenil) propiónico, fue descubierto por el Dr.
Stewart Adams en 1961. Se recomiendo en el manejo de dolor agudo. El efecto terapéutico del ibuprofeno inicia con la unión reversible a los receptores COX (COX1-COX2) al unirse a estos receptores bloquea la unión del ácido araquidónico impidiendo así el paso intermedio para la formación de prostaglandinas, las cuales son responsables del calor, dolor, rubor es decir inflamación. Además, su característica analgésica está relacionada con la sensibilidad que presentan las fibras nerviosas del dolor a este. Existen efectos adversos de su uso crónico; ya que las prostaglandinas y tromboxano mantiene funciones de mantenimiento de la mucosa gástrica y el flujo sanguíneo renal, causan síntomas gastrointestinales como dispepsia incluso en dosis terapéuticas, a nivel renal disminuyen la perfusión de los vasos renales aferentes causando disminución de la tasa de filtración glomerular (13).

- Acetaminofén con codeína: la codeína es un agonista del receptor mu a nivel del SNC, al igual que la morfina. Pertenece a la familia de los opioides, es relativamente débil, se metabolizada en el hígado por el citocromo P450, específicamente a través de la isoenzima CYP2D6 el metabolismo de esta isoenzima varía en la población general, ahí radica lo variable del alivio del dolor al usar la codeína como único agente. Se suele utilizar en combinación con el acetaminofén por el efecto analgésico aditivo (12).

- Opioides: son sustancias derivadas de la adormidera o sus análogos sintéticos. 
Los más reconocidos son: morfina, heroína, tramadol, oxicodona y metadona. Su uso puede crear dependencia, que se caracteriza por un fuerte deseo de consumir la sustancia, deterioro de la capacidad para controlar su uso, consumo persistente de opioides pese a las consecuencias perjudiciales, se da mayor prioridad al consumo y se des enfatizan otras actividades $y$ obligaciones, aumento de la tolerancia y la aparición de síntomas de abstinencia cuando se interrumpe el consumo (16). El tratamiento del dolor intenso, la mejor elección será un opioide de alta potencia como morfina o fentanilo (4). En nuestro sistema de salud los más utilizados son:

- Tramadol: molécula no opioide, agonista selectivo del receptor opioide. Esta droga tiene una potencia analgésica menor respecto a la morfina 1:10. Estabilidad hemodinamica y menor riesgo de depresión respiratoria (4).

- Morfina: es el opioide prototipo. Tarda unos 5 minutos en obtener su efecto analgésico, con su pico a los 15-30 min y duración de 3 a 4 horas por lo que otorga analgesia mantenida más estable (4).

- Fentanilo: opioide sintético de la familia fenilpiperidina, poca reacción cruzada con alergia a la morfina. Tiene paso por la barrera hematoencefálica, dando un efecto clínico rápido en 1-2 minutos lo que confiere un muy buen perfil para aliviar el dolor agudo. Efecto pico a los 5 minutos, su duración es de 0.5- 1 hora, via endovenosa, pero la misma presentación lo permite de manera oral, nebulización y nasal. En adultos mayores se recomienda utilizar la mitad de la dosis (4).

- Lidocaína: es un agente anestésico local de tipo amida que actúa estabilizando las membranas neuronales. Altera la permeabilidad de la membrana al sodio, que a su vez bloquea la propagación de los impulsos y, por lo tanto, amortigua tanto la sensibilización de los nociceptores periféricos como, finalmente, la hiperexcitabilidad del sistema nervioso central. El uso prolongado puede causar la pérdida de fibras nerviosas epidérmicas (14).

Existen varias presentaciones del medicamento, vía oral, pomada, gel, IV y el parche que, además del efecto anestésico local, proporciona protección contra la estimulación mecánica (alodinia dinámica) (14), muy útil en la neuralgia post herpética.

- Midazolam: es una benzodiazepina de acción corta, se utiliza principalmente para la premedicación, sedación, inducción y mantenimiento de la anestesia general. No es utilizado comúnmente en el servicio de urgencias para el manejo del dolor, sin embargo, es fundamental en este servicio para el manejo de sedación (7).

- Bloqueos nerviosos: los bloqueos de nervios periféricos o plexos pueden ser usados cuando el dolor ocurre en el campo de uno o más nervios periféricos, o si el dolor es causado por complicaciones como fracturas 
patológicas u oclusiones vasculares. Son usado como coadyuvante de analgesia sistémica (14). Hay que tener en cuenta que dichos bloqueos pueden producir neuritis, por lo que en pacientes con buen pronóstico no se recomiendan ya que los efectos adversos pueden ser más difíciles de manejar que el dolor original.

\begin{tabular}{|c|}
\hline $\begin{array}{l}\text { Tabla 1. Clasificación de los } \\
\text { antiinflamatorios no esteroideos según su } \\
\text { selectividad para la ciclooxigenasa }\end{array}$ \\
\hline 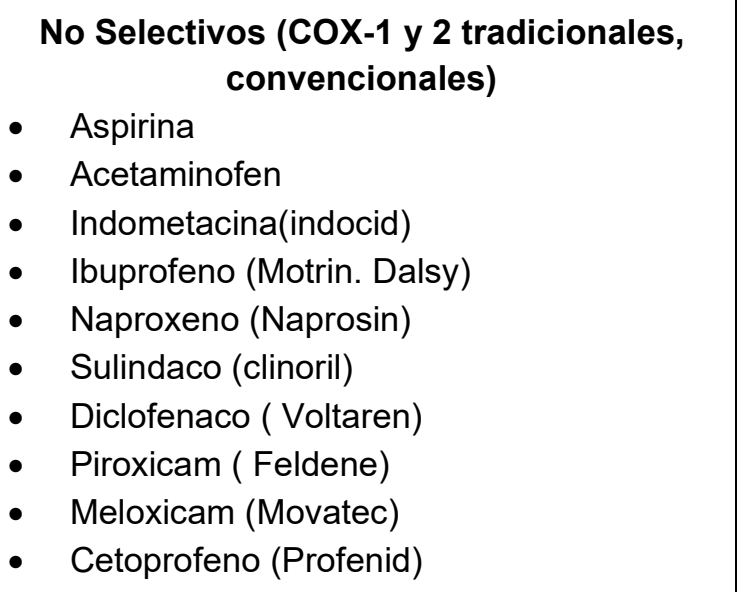 \\
\hline $\begin{array}{ll} & \text { Selectivos (COX-2 COXIBEs) } \\
\text { - } & \text { Rofecoxid (Vioxx) } \\
\text { - } & \text { Valdecoxib (Bextra) } \\
\text { - } & \text { Parecoxib } \\
\text { - } & \text { Celecoxid (celebra) } \\
\text { - } & \text { Etoricoxib ( Arcoxia) } \\
\text { - } & \text { Lumiracoxid (Prexige) } \\
\text { - } & \end{array}$ \\
\hline 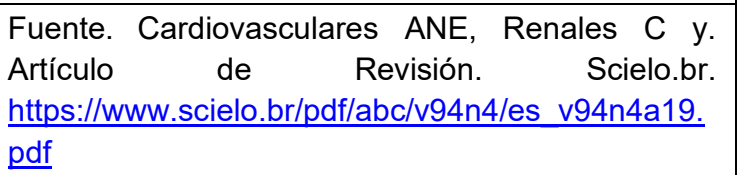 \\
\hline
\end{tabular}

\section{Tratamientos no farmacológicos}

Los tratamientos complementarios como psicoterapia, principalmente la terapia cognitivo conductual y el abordaje a través de terapia física, o bien medicina de rehabilitación deben prescribirse como parte de un enfoque multidisciplinario (13).

Entre los manejos no farmacológicos se debe incluir una adecuada comunicación médico paciente, se puede acompañar con técnicas de intervención física con, hielo o calor, aerosoles refrigerantes tópicos, actividad física, ejercicio adecuado para cada paciente, y técnicas de relajación (9).

Un correcto abordaje con estas modalidades puede mejorar la sintomatología y reducir el riesgo de daños por terapia farmacológica. Es importante recalcar que se necesitan más estudios que examinen el efecto de la terapia conductual en el tratamiento de dolor en pacientes de urgencias, ya que no hay evidencia con respecto a su eficacia en estos pacientes.

\section{CONCLUSIONES}

El dolor actualmente ha sido muy estudiado no solo por su afectación a nivel de salud sino también por el gran impacto socioeconómico que genera a nivel mundial. Es la causa principal de atención en el departamento de urgencias.

Fisiopatológicamente se conocen múltiples mecanismos implicados en este lo cual ha permitido alcanzar un abordaje relativamente optimo en cuanto a dolor agudo en las consultas de urgencias, sin embargo, al hablar del manejo del dolor crónico en el servicio urgencias y el abordaje de sus exacerbaciones, en nuestro sistema de salud, aún existen deficiencias.

Si bien es cierto el dolor es una sensación la cual es percibida de manera negativa, pero al realizar un abordaje rápido, eficaz y de manera multidisciplinaria, utilizando los fármacos que tenemos a disposición de manera adecuada, según el riesgo/beneficio 
en cada paciente de manera individual, además acompañado de las medidas no farmacológicas, de manera conjunta nos permite tener resultados óptimos, generando así una mejor calidad de vida.

\section{Los autores declaran no tener conflicto de interés.}

\section{REFERENCIAS}

1. Sampson FC, O'Cathain A, Goodacre S. How can pain management in the emergency department be improved? Findings from multiple case study analysis of pain management in three UK emergency departments. Emerg Med J. 2020;37(2):85-94.

https://emj.bmj.com/content/37/2/85.abstract

2. Physician credentialing and delineation of clinical privileges in emergency medicine. Ann Emerg Med. 2017;70(3):446.

https://www.acep.org/patient-care/policystatements/physician-credentialing-anddelineation-of-clinical-privileges-in-emergencymedicine/

3. Todd $\mathrm{KH}$. A review of current and emerging approaches to pain management in the emergency department. Pain Ther. 2017;6(2):193-202.

https://pubmed.ncbi.nlm.nih.gov/29127600/

4. García-Andreu J. Manejo básico del dolor agudo y crónico [Internet]. Org.mx. 2017 [cited 2021 Jul 27]. Disponible en: http://www.scielo.org.mx/pdf/am/v29s1/24488771-am-29-00077.pdf

5. León MX, Santa-Cruz JG, Martínez-Rojas S, Ibatá-Bernal L. Recomendaciones basadas en evidencia para el manejo del dolor oncológico (revisión de la literatura). Rev Mex Anest. 2019;42(1):45-55.

https://www.medigraphic.com/cgibin/new/resumen.cgi?IDARTICULO $=84793$

6. Abiuso N, Santelices JL, Quezada R. MANEJO DEL DOLOR AGUDO EN EL SERVICIO DE URGENCIA. Rev médica Clín Las Condes. 2017;28(2):248-60. https://www.elsevier.es/esrevista-revista-medica-clinica-las-condes-202articulo-manejo-del-dolor-agudo-en$\underline{\text { S0716864017300391 }}$

7. Berber MÁR. Evaluación del efecto sedativo del Midazolam con y sin Ketamina, por vía subcutánea, en pacientes dentales pediátricos. Ucr.ac.cr. 27-IX-2017 [cited 2021 Jul 27].
Disponible

en:

https://revistas.ucr.ac.cr/index.php/Odontos/articl e/view/30760/30844\#: :text=Los\%20resultados\% 20del\%20presente $\% 20$ estudio,dental\%20de $\% 201$ os $\% 20$ pacientes $\% 20$ pedi\%C3\%A1tricos

8. Sergey M, Reuben S, Bryan H, Mark R, Steven R, Melanie $\mathrm{R}$, et al. AAEM white paper on acute pain management in the emergency department [Internet]. Aaem.org. 2017 [cited 2021 Jul 27]. Disponible

en: https://www.aaem.org/resources/statements/posit ion/white-paper-on-acute-pain-management-inthe-emergency-department

9. Balakumaran J. Approach to pain management in the ED [Internet]. Canadiem.org. 2020 [cited 2021 Jul 27]. Available from: https://canadiem.org/approach-to-painmanagement-in-the-ed/

10. Neuropathic pain: update in definitions, its pharmacological treatment. Dolor neuropático: actualización en definiciones y su tratamiento farmacológico [Internet]. Bvs.hn. [cited $2021 \mathrm{Jul}$ 27]. Available from: http://www.bvs.hn/RMH/pdf/2020/pdf/Vol88-12020-11.pdf

11. Rincón Carvajal AM, Olaya Osorio CA, Martínez Rojas S, Ibatá Bernal L. Recomendaciones basadas en evidencia para el manejo del dolor neuropático en Atención Primaria en Salud (Revisión de la literatura). Rev Soc Esp Dolor. 2018;25(6):349-58.

https://scielo.isciii.es/scielo.php?script=sci arttext \&pid=S1134-80462018000600349

12. Marchand DK, McCormack S. Codeine for acute pain in patients undergoing orthopedic surgery: A review of clinical effectiveness. Ottawa (ON): Canadian Agency for Drugs and Technologies in Health; 2019. https://www.ncbi.nlm.nih.gov/books/NBK551871/

13. Ershad M, Ameer MA, Vearrier D. Ibuprofen Toxicity. In: StatPearls. Treasure Island (FL): StatPearls $\quad$ Publishing; 2021. https://www.ncbi.nlm.nih.gov/books/NBK526078/

14. Cabo de Villa ED, Morejón Hernández JM, Acosta Figueredo E. Dolor y analgésicos. Algunas consideraciones oportunas. Medisur. 2020;18(4):694-705.

http://scielo.sld.cu/scielo.php?script=sci arttext\& pid $=$ S1727897X2020000400694\#: :text=Administre\%20los \%20analg\%C3\%A9sicos\%20en\%20forma,emple o\%20de\%20ansiol\%C3\%ADticos\%20y\%20antid epresivos 
15. Pérez A, Flores SE, García-Gallont R. Tratamiento multimodal del dolor agudo postoperatorio agregando paracetamol IV a protocolo ya establecido. Rev Fac Med. 2018;1(25):55-63. https://revistamedicina.ufm.edu/publicaciones/wp-

content/uploads/2019/10/Tratamiento-

multimodal-del-dolor-agudo-postoperatorioagregando-paracetamol-IV-a-protocolo-yaestablecido.pdf

16. Carreraa MAV, Correab SMV, Pradoc MMC, Granados KPM. Manejo de intoxicación por opioides [Internet]. Recimundo.com. 2019 [cited 2021 Jul 27]. Disponible en: https://recimundo.com/index.php/es/article/view/6 $\underline{72 / 935}$ 